\title{
A BARRIER OPTION OF AMERICAN TYPE*
}

\author{
IOANNIS KARATZAS \\ Departments of Mathematics \\ and Statistics, 619 Math. Bldg. \\ Columbia University \\ New York, NY 10027 \\ ik@math.columbia.edu
}

\author{
HUI WANG \\ Department of Statistics \\ 618 Mathematics Building \\ Columbia University \\ New York, NY 10027 \\ wanghui@stat. columbia.edu
}

June 2, 2007

\begin{abstract}
We obtain closed-form expressions for the prices and optimal hedging strategies of American put-options in the presence of an "up-and-out" barrier, both with and without constraints on the short-selling of stock. The constrained case leads to a stochastic optimization problem of mixed optimal stopping/singular control type. This is reduced to a variational inequality which is then solved explicitly in two qualitatively separate cases, according to a certain compatibility condition among the market coefficients and the constraint.
\end{abstract}

Key Words: American option, barrier option, singular stochastic control, optimal stopping, variational inequality, hedging, elastic boundary condition, constrained portfolios.

AMS 1991 Subject Classifications: Primary 93E20, 90A09, 60H30; Secondary 60G40, 60G44, $90 \mathrm{~A} 16$.

\footnotetext{
${ }^{*}$ Supported in part by the National Science Foundation under Grant NSF-DMS-97-32810.
} 


\section{INTRODUCTION}

We solve in closed form the pricing and hedging problems for the "up-and-out" Barrier Put-Option of American type, with payoff

$$
Y(t)=(q-S(t))^{+} 1_{\left\{t<\tau_{h}\right\}}, \quad 0 \leq t<\infty .
$$

Here $h>0$ is the barrier and $q \in(0, h)$ the strike-price of the option, whereas

$$
\tau_{h} \triangleq \inf \{t \geq 0 / S(t) \geq h\}
$$

is the time when the option becomes "knocked-out". The stock price-per-share $S(\cdot)$ is assumed to satisfy the standard model

$$
d S(t)=S(t)\left[r d t+\sigma d W_{0}(t)\right], \quad S(0)=x \in(0, h)
$$

of Merton (1973) and Black \& Scholes (1973), with $r>0$ the prevalent interest rate of the risk-free asset (bank account), $\sigma>0$ the volatility of the stock, and $W_{0}(\cdot)$ a Brownian Motion under the risk-neutral equivalent martingale measure.

This analysis is carried out in Section 2. It is shown there that the optimal hedging portfolioweight is always negative, selling the stock short, and decreases without a lower bound as the stock-price approaches the barrier level $h$. As a consequence, the resulting portfolio is rather undesirable from the point of view of practical implementation.

To remedy this situation, we discuss in Section 3 the same problem but now under a shortselling constraint: the hedging portfolio-weight is not allowed to fall below $-\alpha$, for some given constant $\alpha>0$. Using the theory developed by Karatzas \& Kou (1998) for American contingent claims under constraints, we solve this problem also in closed form: first for $\alpha>2 r / \sigma^{2}$ (Section 4) and then for $0<\alpha \leq 2 r / \sigma^{2}$ (Section 5). In the latter case the short-selling constraint is "severe" (cf. Remark 3.4 for discussion) and the price of the option is given by the $\alpha$-enlargement

$$
\phi_{\alpha}(x) \triangleq \sup _{\nu \geq 0}\left[e^{-\alpha \nu} \phi\left(x e^{-\nu}\right)\right]=\left\{\begin{array}{cl}
q-x & ; \quad 0 \leq x \leq \frac{\alpha}{1+\alpha} q \\
\frac{q}{1+\alpha}\left(\frac{\alpha q}{1+\alpha}\right)^{\alpha} x^{-\alpha} & ; \quad \frac{\alpha}{1+\alpha} q<x<\infty
\end{array}\right\}
$$

of the reward function $\phi(x)=(q-x)^{+}$for the American put-option, introduced by Broadie, Cvitanić \& Soner (1998).

In the case $\alpha>2 r / \sigma^{2}$ the short-selling constraint is less severe; the value of the option dominates the quantity of (1.4), and is given by the solution to a stochastic optimization problem that involves both optimal stopping and singular control of the "monotone follower" type, as in Karatzas \& Shreve (1984). We cast this problem in terms of a Variational Inequality, featuring the function $\phi_{\alpha}(\cdot)$ of (1.4), which can then be solved explicitly. The details are carried out in Theorem 4.2 (proved in Appendix A) and in Proposition 4.3, respectively.

The relevance of singular stochastic control to the pricing of European-type barrier options was first brought out in Wystup (1997). An analysis of "down-and-out" barrier call-options, of both European and American type, is carried out in Section 8.9 of Merton (1973). 


\section{AMERICAN PUT-OPTION OF BARRIER TYPE}

Let us consider the standard model of Merton (1973), Black \& Scholes (1973) for a financial market:

$$
\begin{aligned}
d B(t) & =B(t) r d t, \quad B(0)=1 \\
d S(t) & =S(t)[b(t) d t+\sigma d W(t)], \quad S(0)=x>0 .
\end{aligned}
$$

This model consists of a money-market with constant interest rate $r>0$ and price $B(t)=e^{r t}$ (the so-called "numéraire"), and of one stock with price-per-share $S(t)$, constant volatility $\sigma>0$, and appreciation rate $b(t)$ at time $t$. The driving process $W=\{W(t) ; 0 \leq t<\infty\}$ is standard Brownian motion on a probability space $(\Omega, \mathcal{F}, \mathbf{P})$; we shall denote by $\mathbf{F}=\{\mathcal{F}(t)\}_{0 \leq t<\infty}$ the filtration generated by this process, namely $\mathcal{F}(t) \triangleq \sigma(W(s) ; 0 \leq s \leq t)$. It will be assumed that the appreciation rate process $b(\cdot)$ is bounded, and progressively measurable with respect to $\mathbf{F}$.

In such a context, we are interested in the valuation problem for an American put-option of the "up-and-out" barrier type, with payoff

$$
Y(t)=(q-S(t))^{+} 1_{\left\{t<\tau_{h}\right\}}, \quad 0 \leq t<\infty .
$$

To place this in context, imagine a contract, signed at time $t=0$, which confers to its holder the right (but not the obligation, whence the term "option") to sell to the issuer one share of the stock, at the contractually specified price $q>0$ and at any time of the holder's choice, provided that a (contractually specified) barrier $h \in(q, \infty)$ has not yet been reached or exceeded. In other words, the contract is "knocked-out" for (i.e., becomes worthless to) its holder at the first time

$$
\tau_{h} \triangleq \inf \{t \geq 0 / S(t) \geq h\}
$$

the stock-price $S(\cdot)$ reaches or exceeds the barrier level $h$. Clearly, if the holder of the contract exercises his option at time $t$, then effectively he receives from the issuer a payment of size $Y(t) \geq 0$ as in (2.2). Such a contract is of potential value to a holder who believes that the stock-price will fall below $q$, and to an issuer who believes otherwise but does not want to have to worry about hedging if the stock-price should become too high (i.e., reach or exceed the barrier $h$ ).

How much should then the issuer charge his counterpart at $t=0$, for signing this contract? In other words, what is the price, at time $t=0$, of the American contingent claim $Y(\cdot)$ in (2.2), (2.3)? From the standard theory on American contingent claims (e.g. Karatzas (1996), §1.4) we know that this so-called hedging price is defined as the smallest initial captial $\xi>0$ that allows the issuer to cover his obligation successfully (i.e., without risk), no matter when the holder should decide to exercise his option:

$$
H(x) \triangleq \inf \left\{\xi>0 / \exists(\pi, C) \text { with } X^{\xi, \pi, C}(\tau) \geq Y(\tau) \quad(\forall \tau \in \mathcal{S})\right\} .
$$

Here $\mathcal{S}$ is the class of all $\mathbf{F}$-stopping times; $\pi(\cdot)$ is a portfolio process (F-progressively measurable, with $\int_{0}^{T} \pi^{2}(t) d t<\infty$ a.s., for any $\left.0<T<\infty\right)$; and $C(\cdot)$ is a cumulative consumption process (measurable and $\mathbf{F}$-adapted, with values in $[0, \infty)$ and increasing, right continuous paths with $C(0)=0$ a.s.). Finally, $X(\cdot) \equiv X^{\xi, \pi, C}(\cdot)$ is the wealth process corresponding to initial capital $\xi$, portfolio $\pi(\cdot)$ and cumulative consumption $C(\cdot)$, namely

$$
d X(t)=\pi(t)\left[r d t+\sigma d W_{0}(t)\right]+(X(t)-\pi(t)) r d t-d C(t), \quad X(0)=\xi,
$$


or equivalently

$$
e^{-r t} X^{\xi, \pi, C}(t)=\xi-\int_{(0, t]} e^{-r s} d C(s)+\sigma \int_{0}^{t} e^{-r s} \pi(s) d W_{0}(s), \quad 0 \leq t<\infty,
$$

where

$$
W_{0}(t) \triangleq W(t)+\int_{0}^{t} \frac{b(s)-r}{\sigma} d s, \quad 0 \leq t<\infty .
$$

From this same general theory, we also know that the hedging price $H(x)$ of $(2.4)$ can be computed as the optimal expected reward in a problem of optimal stopping:

$$
H(x)=G(x) \triangleq \sup _{\tau \in \mathcal{S}} \mathbf{E}_{0}\left[e^{-r \tau}(q-S(\tau))^{+} 1_{\left\{\tau<\tau_{h}\right\}}\right]=\sup _{\tau \in \mathcal{S}} \mathbf{E}_{0}\left[e^{-r \tau}(q-S(\tau))^{+} 1_{\left\{\max _{0 \leq t \leq \tau} S(t)<h\right\}}\right] .
$$

We have denoted by $\mathbf{E}_{0}$ the expectation under the probability measure $\mathbf{P}_{0}$ on $(\Omega, \mathcal{F})$ with

$$
\left.\frac{d \mathbf{P}_{0}}{d \mathbf{P}}\right|_{\mathcal{F}(t)}=\exp \left\{-\int_{0}^{t}\left(\frac{b(s)-r}{\sigma}\right) d W(s)-\frac{1}{2} \int_{0}^{t}\left(\frac{b(s)-r}{\sigma}\right)^{2} d s\right]
$$

for every $t \in(0, \infty)$. Under this measure $\mathbf{P}_{0}$, the process $W_{0}(\cdot)$ of $(2.6)$ is standard Brownian motion by the Girsanov theorem, and we may rewrite (2.1) in the form of (1.3).

2.1 REMARK: We refer the reader to the discussion on pp. 192-193 in Karatzas \& Shreve (1991) for the Girsanov theorem on an infinite time-horizon, and to $\S \S 1.7,2.6$ in Karatzas \& Shreve (1998) for the measure-theoretic subtleties associated with questions of hedging on an infinite time-horizon.

How are we then to solve the optimal stopping problem of (2.7)? By analogy with Example 1.4.8 in Karatzas (1996), we cast this problem as a variational inequality. For an alternative approach, that does not rely on the so-called "principle of smooth-fit", the reader may wish to consult and apply the results of Salminen (1985).

2.2 VARIATIONAL InEQUALITY: Find a number $b \in(0, q)$ and a convex, decreasing function $g(\cdot)$ in the space $\mathcal{C}([0, \infty)) \cap \mathcal{C}_{b}^{1}((0, \infty) \backslash\{h\}) \cap \mathcal{C}_{b}^{2}((0, \infty) \backslash\{b, h\})$, such that:

$$
\begin{aligned}
\frac{\sigma^{2}}{2} x^{2} g^{\prime \prime}(x)+r x g^{\prime}(x)-r g(x)=0 & ; \quad b<x<h \\
\frac{\sigma^{2}}{2} x^{2} g^{\prime \prime}(x)+r x g^{\prime}(x)-r g(x)<0 & ; \quad 0<x<b \\
g(x)>(q-x)^{+} & ; \quad x>b \\
g(x)=(q-x)^{+} & ; \quad 0 \leq x \leq b \\
g(x)=0 & ; \quad h \leq x<\infty .
\end{aligned}
$$

2.3 THEOREM: If the pair $(b, g(\cdot))$ solves the Variational Inequality 2.2, then $g(\cdot)$ coincides with the optimal expected reward of the stopping problem in (2.7), and the stopping time

$$
\tau_{b} \triangleq \inf \{t \geq 0 / S(t) \leq b\}
$$


is optimal for this problem:

$$
g(x)=G(x)=\sup _{\tau \in \mathcal{S}} \mathbf{E}_{0}\left[e^{-r \tau}(q-S(\tau))^{+} 1_{\left\{\tau<\tau_{h}\right\}}\right]=\mathbf{E}_{0}\left[e^{-r \tau_{b}}\left(q-S\left(\tau_{b}\right)\right)^{+} 1_{\left\{\tau_{b}<\tau_{h}\right\}}\right] .
$$

Proof: All claims are obvious for $x \geq h$, so let us concentrate on a fixed $x \in(0, h)$. On the interval $(0, h)$, the functions $g(\cdot), g^{\prime}(\cdot)$ are bounded, continuous; and the function $g^{\prime \prime}(\cdot)$ is bounded, continuous on $(0, h) \backslash\{b\}$. An application of Itô's rule (cf. Problem 3.7.3, p. 219 in Karatzas \& Shreve (1991)) to the process $e^{-r t} g(S(t)), 0 \leq t<\infty$ yields then, in conjunction with (2.8)-(2.10):

$$
\begin{aligned}
& g(x)-e^{-r\left(\tau \wedge \tau_{h}\right)} g\left(S\left(\tau \wedge \tau_{h}\right)\right)+\left.\sigma \int_{0}^{\tau \wedge \tau_{h}} e^{-r t}\left(\xi g^{\prime}(\xi)\right)\right|_{\xi=S(t)} d W_{0}(t) \\
= & -\left.\int_{0}^{\tau \wedge \tau_{h}} e^{-r t}\left(\frac{\sigma^{2}}{2} \xi^{2} g^{\prime \prime}(\xi)+r \xi g^{\prime}(\xi)-r g(\xi)\right)\right|_{\xi=S(t)} d t \geq 0, \quad \mathbf{P}_{0}-\text { a.s. }
\end{aligned}
$$

for every $\tau \in \mathcal{S}$. Now the $\mathbf{P}_{0}$-expectation of the stochastic integral in (2.16) is equal to zero; indeed, from the convexity and decrease of $g(\cdot)$ we have $0 \leq-\xi g^{\prime}(\xi) \leq g(0)-g(\xi) \leq g(0)$ for $0<\xi<\infty$, and thus $\mathbf{E}_{0} \int_{0}^{\infty} e^{-2 r t}\left(S(t) g^{\prime}(S(t))\right)^{2} d t \leq g^{2}(0) / 2 r<\infty$. We obtain

$$
\begin{aligned}
g(x) & \geq \mathbf{E}_{0}\left[e^{-r\left(\tau \wedge \tau_{h}\right)} g\left(S\left(\tau \wedge \tau_{h}\right)\right)\right] \\
& =\mathbf{E}_{0}\left[e^{-r \tau} g(S(\tau)) 1_{\left\{\tau<\tau_{h}\right\}}\right]+\mathbf{E}_{0}\left[e^{-r \tau_{h}} g\left(S\left(\tau_{h}\right)\right) 1_{\left\{\tau_{h} \leq \tau, \tau_{h}<\infty\right\}}\right] \\
& =\mathbf{E}_{0}\left[e^{-r \tau} g(S(\tau)) 1_{\left\{\tau<\tau_{h}\right\}}\right] \\
& \geq \mathbf{E}_{0}\left[e^{-r \tau}(q-S(\tau))^{+} 1_{\left\{\tau<\tau_{h}\right\}}\right]
\end{aligned}
$$

from (2.11)-(2.13); in other words, $g(x) \geq G(x)$.

On the other hand, thanks to (2.9) and (2.12), all the inequalities in (2.16), (2.17) hold as equalities for the choice $\tau \equiv \tau_{b}$ of (2.14). The claims of (2.15) now follow readily.

It remains to construct the solution of the Variational Inequality 2.2.

2.4 Proposition: Let $b$ be the unique solution of the equation

$$
1+\beta\left(\frac{b}{q}\right)=\beta+\left(\frac{b}{h}\right)^{\beta}
$$

in the interval $(0, q)$, where $\beta \triangleq 1+\left(2 r / \sigma^{2}\right)$, and define

$$
g(x) \triangleq\left\{\begin{array}{ccc}
q-x & ; & 0 \leq x \leq b \\
x\left(\frac{q-b}{b}\right) \frac{(h / x)^{\beta}-1}{(h / b)^{\beta}-1} & ; \quad b<x<h \\
0 & ; \quad h \leq x<\infty
\end{array}\right\} .
$$

Then the pair $(b, g(\cdot))$ solves the Variational Inequality 2.2 .

Proof: The general solution of the equation (2.9) is given by $g(x)=A x^{-\gamma_{+}}+B x^{-\gamma_{-}}$for suitable constants $A, B$, where $\gamma_{+}=2 r / \sigma^{2}, \gamma_{-}=-1$ are the roots of $\sigma^{2} \gamma(\gamma+1) / 2-r \gamma-r=0$. The requirements $g(h-)=0, g(b+)=q-b$ (continuity of $g(\cdot)$ at the points $x=h$ and $x=b$ ) 
and $g^{\prime}(b+)=-1$ (continuity of $g^{\prime}(\cdot)$ at the point $x=b$; principle of smooth-fit) lead then to the equation (2.18) for $b$, and to the formulae $A=[(q / b)-1]\left(b^{-\beta}-h^{-\beta}\right)^{-1}, B=-A h^{-\beta}$ for the two constants. These, in turn, yield the expression of (2.19).

In order to see that $b \in(0, q)$ is determined uniquely by the equation $(2.18)$, note that

$$
F(u) \triangleq \beta-1+\left(\frac{u}{h}\right)^{\beta}-\beta\left(\frac{u}{q}\right), \quad 0 \leq u<\infty
$$

is convex, with $F(0)=\beta-1>0, \quad F(q)=(q / h)^{\beta}-1<0$. Thus $F(\cdot)$ has exactly one root in the interval $(0, q)$. Furthermore, notice from (2.19) that

$$
-x g^{\prime}(x)=\left\{\begin{array}{ccc}
x & ; & 0<x<b \\
x\left(\frac{q-b}{b}\right) \frac{\gamma_{+}(h / x)^{\beta}+1}{(h / b)^{\beta}-1} & ; \quad b<x<h \\
0 & ; \quad h<x<\infty
\end{array}\right\} .
$$

In the interval $(0, b)$, we have $\frac{\sigma^{2}}{2} x^{2} g^{\prime \prime}(x)+r x g^{\prime}(x)-r g(x)=-r q<0$; in the interval $(b, h)$ we have $g^{\prime}(\cdot)<0$, since $1+\gamma_{+}(h / x)^{\beta}>1+\gamma_{+}=\beta>0$. In other words, $g(\cdot)$ is strictly decreasing on $(0, h)$. Furthermore, by (2.19) and (2.20), we have

$$
\frac{\sigma^{2}}{2} x^{2} g^{\prime \prime}(x)=r\left[g(x)-x g^{\prime}(x)\right]>0, \quad b<x<h
$$

and thus $g(\cdot)$ is strictly convex on $(b, h)$. It is now clear that the pair $(b, g(\cdot))$ solves the Variational Inequality 2.2 .

The theory of $\S 1.4$ in Karatzas (1996) also provides the optimal hedging portfolio process $\hat{\pi}(\cdot)$ and the cumulative consumption (or "cash-flow") process $\hat{C}(\cdot)$, in the form

$$
\begin{aligned}
\hat{\pi}(t) & =\left\{\begin{array}{cc}
\left.x g^{\prime}(x)\right|_{x=S(t)} \quad ; \quad 0 \leq t<\tau_{h} \\
0 \quad & \tau_{h} \leq t<\infty
\end{array}\right\} \\
\hat{C}(t) & =-\left.\int_{0}^{t \wedge \tau_{h}}\left(\frac{\sigma^{2}}{2} x^{2} g^{\prime \prime}(x)+r x g^{\prime}(x)-r g(x)\right) 1_{(0, b)}(x)\right|_{x=S(u)} d u \\
& =r q \int_{0}^{t \wedge \tau_{h}} 1_{(0, b)}(S(u)) d u, \quad 0 \leq t<\infty .
\end{aligned}
$$

The corresponding value process is also given explicitly, by

$$
X^{g(x), \hat{\pi}, \hat{C}}(t) \equiv \hat{X}(t)=\left\{\begin{array}{cll}
g(S(t)) & ; \quad 0 \leq t<\tau_{h} \\
0 & ; \quad \tau_{h} \leq t<\infty
\end{array}\right\}
$$

In particular,

$$
\hat{p}(t) \triangleq\left\{\begin{array}{cc}
\hat{\pi}(t) / \hat{X}(t)=\left.\left(\frac{x g^{\prime}(x)}{g(x)}\right)\right|_{x=S(t)} & ; \quad 0 \leq t<\tau_{h} \\
0 & ; \quad \tau_{h} \leq t<\infty
\end{array}\right\}
$$

are the optimal hedging portfolio-weights, or proportions. Finally, the stopping time $\tau_{b}$ of (2.14) is the optimal exercise time for this American put-option of "up-and-out" barrier type, by its holder. 
From (2.19), (2.20) and (2.24), we notice that the "leverage ratio"

$$
\frac{x g^{\prime}(x)}{g(x)}=1-\frac{\beta}{1-(x / h)^{\beta}}, \quad b<x<h
$$

decreases without bound (i.e., down to $-\infty$ ), as $x \uparrow h$. In other words, as the stock price approaches the barrier $h$, the optimal hedging portfolio sells short vast amounts of stock, measured in units of the option's value.

Such a portfolio is rather undesirable, from the point of view of practical implementation. In order to get around this problem, we shall impose throughout the remainder of this paper restrictions on the short-selling of stock, in the form of constraints on the portfolio-weights $p(t)=$ $\pi(t) / X(t)$.

\section{CONSTRAINED SHORT-SELLING}

Suppose now that we decide to constrain the short-selling of stock, by requiring that the portfolioweight

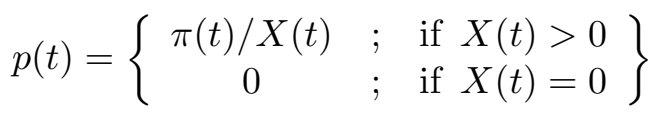

(i.e., the proportion of the wealth $X(t)$ that is invested in stock) should always exceed a given lower bound $-\alpha$. In other words, we impose the leverage constraint

$$
\pi(t) \geq-\alpha X^{\xi, \pi, C}(t), \quad \forall 0 \leq t<\infty
$$

for some given real constant $\alpha>0$. We saw at the end of the last section that such a requirement is in fact desirable from a practical point of view, when one is trying to hedge the American put-option of barrier type.

How does this requirement affect the hedging price of the American barrier option of (2.2)-(2.3)? In other words, what is the analogue

$$
H_{\alpha}(x) \triangleq \inf \left\{\xi>0 / \exists(\pi, C) \text { s.t. }(3.2) \text { holds, and } X^{\xi, \pi, C}(\tau) \geq Y(\tau) \quad(\forall \tau \in \mathcal{S})\right\}
$$

of (2.4) under this new, additional constraint?

In general terms, the answer to this question is provided by the theory developed in Karatzas \& Kou (1998); it is shown there that $H_{\alpha}(x)$ can be computed, in principle, in terms of a double stochastic optimization problem

$$
H_{\alpha}(x)=G_{\alpha}(x) \triangleq \sup _{\lambda(\cdot) \in \mathcal{D}} \sup _{\tau \in \mathcal{S}} \mathbf{E}_{\lambda}\left[e^{-\int_{0}^{\tau}(r+\delta(\lambda(t))) d t}(q-S(\tau))^{+} \cdot 1_{\left\{\max _{0 \leq t \leq \tau} S(t)<h\right\}}\right]
$$

of mixed stochastic control/optimal stopping type. In the notation of that paper, $K=[-\alpha, \infty)$ is the interval where the portfolio-weight process of (3.1) is constrained to take values; and

$$
\delta(\nu) \triangleq \sup _{p \in K}(-p \nu)=\left\{\begin{array}{cc}
\alpha \nu & ; \quad \text { for } \nu \geq 0 \\
\infty & ; \text { for } \nu<0
\end{array}\right\}
$$


is the support function of the set $-K=(-\infty, \alpha]$. We shall denote by $\tilde{K}=\{\nu / \delta(\nu)<\infty\}=[0, \infty)$ the effective domain of $\delta(\cdot)$, and by $\mathcal{D}$ the space of all bounded, $\mathbf{F}$-progressively measurable processes $\lambda(\cdot):[0, \infty) \times \Omega \rightarrow \tilde{K}=[0, \infty)$. Finally, for every $\lambda(\cdot) \in \mathcal{D}, \mathbf{E}_{\lambda}$ denotes expectation with respect to the probability measure $\mathbf{P}_{\lambda}$ with

$$
\left.\frac{d \mathbf{P}_{\lambda}}{d \mathbf{P}}\right|_{\mathcal{F}(t)}=Z_{\lambda}(t) \triangleq \exp \left\{-\int_{0}^{t} \frac{b(u)-r+\lambda(u)}{\sigma} d W(u)-\frac{1}{2} \int_{0}^{t}\left(\frac{b(u)-r+\lambda(u)}{\sigma}\right)^{2} d u\right\}
$$

for $0 \leq t<\infty$.

3.1 REMARK: For any $\lambda(\cdot) \in \mathcal{D}$ and with $\Lambda(\cdot) \triangleq \int_{0}^{\cdot} \lambda(u) d u$, the process

$$
W_{\lambda}(t) \triangleq W(t)+\int_{0}^{t} \frac{b(s)-r+\lambda(s)}{\sigma} d s=W_{0}(t)+\sigma^{-1} \Lambda(t), \quad 0 \leq t<\infty
$$

is Brownian motion under the probability measure $\mathbf{P}_{\lambda}$ of (3.6). Furthermore, (1.3) shows that we have

$$
d S(t)=S(t)\left[(r-\lambda(t)) d t+\sigma d W_{\lambda}(t)\right]=S(t)\left[r d t+\sigma d W_{\lambda}(t)-d \Lambda(t)\right], \quad S(0)=x
$$

and we may write (3.8) in the equivalent form

$$
d S_{\lambda}(t)=S_{\lambda}(t)\left[r d t+\sigma d W_{\lambda}(t)\right], \quad S_{\lambda}(0)=x
$$

for the process

$$
S_{\lambda}(\cdot) \triangleq S(\cdot) e^{\Lambda(\cdot)}
$$

Comparing (3.9) with (1.3), we see that the process $S_{\lambda}(\cdot)$ of (3.10) has the same law under $\mathbf{P}_{\lambda}$, as the law of the original stock-price process $S(\cdot)$ under $\mathbf{P}_{0}$. This suggests that the quantity

$$
G_{\alpha}(x)=\sup _{\lambda(\cdot) \in \mathcal{D}} \sup _{\tau \in \mathcal{S}} \mathbf{E}_{\lambda}\left[e^{-(r \tau+\alpha \Lambda(\tau))}\left(q-e^{-\Lambda(\tau)} S_{\lambda}(\tau)\right)^{+} \cdot 1_{\left\{\max _{0 \leq t \leq \tau}\left(e^{-\Lambda(t)} S_{\lambda}(t)\right)<h\right\}}\right]
$$

of (3.4), might be written equivalently as

$$
G_{\alpha}(x)=\sup _{\lambda(\cdot) \in \mathcal{D}} \sup _{\tau \in \mathcal{S}} \mathbf{E}_{0}\left[e^{-(r \tau+\alpha \Lambda(\tau))}\left(q-e^{-\Lambda(\tau)} S(\tau)\right)^{+} \cdot 1_{\left\{\max _{0 \leq t \leq \tau}\left(e^{-\Lambda(t)} S(t)\right)<h\right\}}\right] .
$$

We shall verify this later, in the proofs for Theorems 4.2 and 5.1 below. The processes $\lambda(\cdot)$ of $\mathcal{D}$ play the role of "Lagrange multipliers", that enforce the short-selling constraint of (3.2). It turns out that the supremum over $\lambda(\cdot) \in \mathcal{D}$ in (3.11) is not attained; the supremum is attained, however, if one enlarges the class $\mathcal{D}$ in the manner of Wystup (1997). More precisely, consider the space $\mathcal{L}^{+}$ of $\mathbf{F}$-adapted processes $\Lambda:[0, \infty) \times \Omega \rightarrow[0, \infty)$ with continuous, increasing paths and $\Lambda(0)=0$. Then, as we shall see in Appendix B and in Lemma 5.2, we can take the supremum in (3.11)' over this larger class without increasing its value: 


$$
G_{\alpha}(x)=\sup _{\Lambda(\cdot) \in \mathcal{L}^{+}} \sup _{\tau \in \mathcal{S}} \mathbf{E}_{0}\left[e^{-(r \tau+\alpha \Lambda(\tau))}\left(q-P_{\Lambda}(\tau)\right)^{+} \cdot 1_{\left\{\max _{0 \leq t \leq \tau} P_{\Lambda}(t)<h\right\}}\right]
$$

We have denoted by

$$
P_{\Lambda}(\cdot) \triangleq e^{-\Lambda(\cdot)} S(\cdot)
$$

the " $\Lambda(\cdot)$-reduced stock price process", which satisfies the equation

$$
d P_{\Lambda}(t)=P_{\Lambda}(t)\left[r d t+\sigma d W_{0}(t)-d \Lambda(t)\right], \quad P_{\Lambda}(0)=x
$$

It should be noted that the resulting double stochastic optimization problem in $(3.11)^{\prime \prime}$ involves both optimal stopping, and singular stochastic control of the monotone-follower type.

3.2 REMARK : For a given $\Lambda(\cdot) \in \mathcal{L}^{+}$, the second maximization in $(3.11)^{\prime \prime}$ amounts to solving an optimal stopping problem analogous to that of (2.7), but with new, reduced numéraire $B_{\Lambda}(t)=e^{-r t-\alpha \Lambda(t)}$ and stock-price process $P_{\Lambda}(\cdot)$ as in $(3.12)$, in place of of $B(\cdot)$ and $S(\cdot)$, respectively. Subjecting the reduced process $P_{\Lambda}(\cdot)$ to the barrier $h>0$, is the same as "raising the barrier" for the original stock-price process $S(\cdot)$ to the new height $h e^{\Lambda}(\cdot)$, which is both time-varying and random. The purpose of the first maximization in $(3.11)^{\prime \prime}$, is to select a $\Lambda^{*}(\cdot) \in \mathcal{L}^{+}$that leads to a raised-barrier $h e^{\Lambda^{*}(\cdot)}$ which is "just right" for computing the quantity $H_{\alpha}(x)$ in $(3.3),(3.4)$.

3.3 REMARK : We can identify a process $\Lambda^{*}(\cdot) \in \mathcal{L}^{+}$that attains the supremum over $\Lambda(\cdot) \in \mathcal{L}^{+}$ in $(3.11)^{\prime \prime}$, as follows:

$$
\Lambda^{*}(t)=\max _{0 \leq u \leq t}\left(\log \left(\frac{S(u)}{h}\right)\right)^{+}, \quad 0 \leq t<\infty
$$

This is the smallest of all processes $\Lambda(\cdot) \in \mathcal{L}^{+}$with the property $P_{\Lambda}(t) \leq h, \forall 0 \leq t<\infty$; in particular,

$$
\int_{0}^{\infty}\left(h-P_{\Lambda^{*}}(t)\right) d \Lambda^{*}(t)=0 .
$$

In other words, the process $\Lambda^{*}(\cdot)$ of (3.14) reduces (pushes to the left, in the stochastic equation (3.13)) the stock-price $S(\cdot)$, only just enough to prevent the resulting process $P_{\Lambda^{*}}(\cdot)$ of $(3.12)$ from ever crossing the barrier $h$. The paths of $\Lambda^{*}(\cdot)$ are continuous, but singular with respect to Lebesgue measure. As we shall see in Theorems 4.2 and 5.1 below, this process $\Lambda^{*}(\cdot)$ attains the supremum in $(3.11)^{\prime \prime}$, namely

$$
G_{\alpha}(x)=\sup _{\tau \in \mathcal{S}} \mathbf{E}_{0}\left[e^{-\left(r \tau+\alpha \Lambda^{*}(\tau)\right)}\left(q-P_{\Lambda^{*}}(\tau)\right)^{+} \cdot 1_{\left\{\max _{0 \leq t \leq \tau} P_{\Lambda^{*}}(t)<h\right\}}\right] .
$$

Notice that this last problem is one of Optimal Stopping for the process $P_{\Lambda^{*}}(t)$ of $(3.12)-(3.15)$. In the next section we shall solve this optimal stopping problem by associating with it a Variational Inequality, similar to that of $(2.9)-(2.13)$.

3.4 REMARK : The expression of (2.25) for the unconstrained-optimal leverage ratio, suggests 
$\alpha_{*}=2 r / \sigma^{2}$ as a critical level for the constant $\alpha>0$ in (3.2), in the sense that for $\alpha \leq \alpha_{*}$ the unconstrained-optimal portfolio $\hat{\pi}(\cdot)$ of $(2.21)$ violates the constraint $(3.2)$ for every $t \in\left[0, \tau_{h}\right)$, or equivalently that

$$
\frac{x g^{\prime}(x)}{g(x)}<-\alpha_{*} \text { holds for every stock-price level } x \in(0, h)
$$

In other words, a constraint of the type (3.2) with $\alpha \leq 2 r / \sigma^{2}$ is "severe", whereas $\alpha>2 r / \sigma^{2}$ can be construed as "mild". These two cases will be discussed separately (in Sections 5 and 4 , respectively), as the solution of the stochastic optimization problem of (3.4) exhibits qualitatively distinct features in each one of them.

\section{A VARIATIONAL INEQUALITY}

By analogy with Theorem 2.3 and $(2.21)-(2.24)$, let us postulate the existence of a function $g_{\alpha}(\cdot)$ and of a process $\Lambda_{\alpha}(\cdot)$ in $\mathcal{L}^{+}$, such that we have

$$
\hat{\pi}_{\alpha}(t)=\left.e^{-\alpha \Lambda_{\alpha}(t)}\left(x g_{\alpha}^{\prime}(x)\right)\right|_{x=P_{\Lambda_{\alpha}}(t)}, \quad \hat{X}_{\alpha}(t) \equiv \hat{X}^{g_{\alpha}(x), \hat{\pi}_{\alpha}, \hat{C}_{\alpha}}(t)=e^{-\alpha \Lambda_{\alpha}(t)} g\left(P_{\Lambda_{\alpha}}(t)\right)
$$

for the optimal hedging portfolio/consumption process pair $\left(\hat{\pi}_{\alpha}(\cdot), \hat{C}_{\alpha}(\cdot)\right)$ and their corresponding value-process $\hat{X}_{\alpha}(\cdot)$, under the constraint (3.2) on the short-selling of stock. In order for this constraint to hold, the function $g_{\alpha}(\cdot)$ must satisfy

$$
\alpha g_{\alpha}(x)+x g_{\alpha}^{\prime}(x) \geq 0, \quad \text { on }(0, h) .
$$

On the other hand, the function $g_{\alpha}(\cdot)$ must dominate on the interval $[0, h)$ the " $\alpha$-enlargement" $\phi_{\alpha}(\cdot)$ of the reward function $\phi(x) \triangleq(q-x)^{+}$, namely

$$
g_{\alpha}(x) \geq \phi_{\alpha}(x), \quad \forall x \in[0, h),
$$

where

$$
\begin{aligned}
& \phi_{\alpha}(x) \triangleq \sup _{\nu \in \tilde{K}}\left[e^{-\delta(\nu)} \phi\left(x e^{-\nu}\right)\right]=\sup _{\nu \geq 0}\left[e^{-\alpha \nu}\left(q-x e^{-\nu}\right)^{+}\right] \\
& =\left\{\begin{array}{cl}
q-x & ; \quad 0 \leq x \leq \frac{\alpha q}{1+\alpha} \\
\frac{q}{1+\alpha}\left(\frac{\alpha q}{1+\alpha}\right)^{\alpha} x^{-\alpha} & ; \quad \frac{\alpha q}{1+\alpha}<x<\infty
\end{array}\right\}
\end{aligned}
$$

is the function of Broadie, Critanić \& Soner (1997); see also Karatzas \& Kou (1998) and Wystup (1997) for other applications of this enlargement. We are thus led to a variational inequality similar to that of Section 2.

4.1 VARIATIONAL INEQUALITY : Find a number $b_{\alpha} \in\left(0, \frac{\alpha q}{1+\alpha}\right)$ and a convex, decreasing func- 
tion $g_{\alpha}(\cdot)$ in the space $\mathcal{C}([0, h)) \cap \mathcal{C}_{b}^{1}((0, h)) \cap \mathcal{C}_{b}^{2}\left((0, h) \backslash\left\{b_{\alpha}\right\}\right)$, such that

$$
\begin{array}{rc}
\frac{\sigma^{2}}{2} x^{2} g_{\alpha}^{\prime \prime}(x)+r x g_{\alpha}^{\prime}(x)-r g_{\alpha}(x)=0 & ; \quad b_{\alpha}<x<h \\
\frac{\sigma^{2}}{2} x^{2} g_{\alpha}^{\prime \prime}(x)+r x g_{\alpha}^{\prime}(x)-r g_{\alpha}(x)<0 & ; 0<x<b_{\alpha} \\
g_{\alpha}(x)>\phi_{\alpha}(x) & ; \quad b_{\alpha}<x<h \\
g_{\alpha}(x)=\phi_{\alpha}(x) \quad ; \quad 0 \leq x \leq b_{\alpha} \\
\alpha g_{\alpha}(x)+x g_{\alpha}^{\prime}(x)>0 \quad ; 0<x<h \\
\alpha g_{\alpha}(h-)+h g_{\alpha}^{\prime}(h-)=0 & \\
g_{\alpha}(x)=0 & ; \quad h \leq x<\infty .
\end{array}
$$

The condition (4.9) suggests that $h>0$ is an "elastic barrier" for the process $P_{\Lambda^{*}}(\cdot)$ of $(3.12)$ (3.15); see section 6.4 in Karatzas \& Shreve (1991) for a study of elastic boundary conditions for Brownian motion.

4.2 THEOREM: If the pair $\left(b_{\alpha}, g_{\alpha}(\cdot)\right)$ solves the Variational Inequality 4.1, then $g_{\alpha}(\cdot)$ coincides with the optimal expected reward in the stopping problem of $(3.11)^{\prime \prime \prime}$, and the stopping time

$$
\tau_{b_{\alpha}}^{*} \triangleq \inf \left\{t \geq 0 / P_{\Lambda^{*}}(t) \leq b_{\alpha}\right\}
$$

attains the supremum in (3.11) $)^{\prime \prime \prime}$. More specifically,

$$
\begin{aligned}
G_{\alpha}(x)=g_{\alpha}(x) & =\mathbf{E}_{0}\left[e^{-r \tau_{b_{\alpha}}^{*}-\alpha \Lambda^{*}\left(\tau_{b_{\alpha}}^{*}\right)}\left(q-P_{\Lambda^{*}}\left(\tau_{b_{\alpha}}^{*}\right)\right)^{+} 1_{\left\{\tau_{b_{\alpha}}^{*}<\tau_{h}^{*}\right\}}\right] \\
& =\sup _{\tau \in \mathcal{S}} \mathbf{E}_{0}\left[e^{-r \tau-\alpha \Lambda^{*}(\tau)}\left(q-P_{\Lambda^{*}}(\tau)\right)^{+} 1_{\left\{\tau<\tau_{h}^{*}\right\}}\right] \\
& =\sup _{\Lambda(\cdot) \in \mathcal{L}^{+}} \sup _{\tau \in \mathcal{S}} \mathbf{E}_{0}\left[e^{-r \tau-\alpha \Lambda(\tau)}\left(q-P_{\Lambda}(\tau)\right)^{+} 1_{\left\{\tau<\tau_{h}^{\Lambda}\right\}}\right]
\end{aligned}
$$

with the notation of (3.14) and

$$
\tau_{h}^{\Lambda} \triangleq \inf \left\{t \geq 0 / P_{\Lambda}(t)>h\right\}, \quad \tau_{h}^{*} \equiv \tau_{h}^{\Lambda^{*}} .
$$

Furthermore, we can replace in the expressions of (4.12) the function $(q-\cdot)^{+}=\phi(\cdot)$ by its $\alpha$ enlargement $\phi_{\alpha}(\cdot)$ of $(4.3)$.

The proof of this result is deferred to Appendix A. We present now the solution of the Variational Inequality 4.1 , in the case $\alpha>2 r / \sigma^{2}$ (of a "mild constraint"). The case $0<\alpha \leq 2 r / \sigma^{2}$ (of a "severe constraint") is treated in the next section.

4.3 Proposition: Suppose that $\alpha>2 r / \sigma^{2}$. With the notation $\beta=1+\left(2 r / \sigma^{2}\right)$ of Proposition 2.4, denote by $b_{\alpha}$ the unique solution of the equation

$$
1+\beta\left(\frac{b_{\alpha}}{q}\right)=\beta+\left(1-\frac{\beta}{1+\alpha}\right)\left(\frac{b_{\alpha}}{h}\right)^{\beta}
$$


in the interval $\left(0, \frac{\alpha q}{1+\alpha}\right)$, and define

$$
g_{\alpha}(x) \triangleq\left\{\begin{array}{ccc}
q-x & ; & 0 \leq x \leq b_{\alpha} \\
x\left(\frac{q-b_{\alpha}}{b_{\alpha}}\right) \frac{(h / x)^{\beta}-1+\frac{\beta}{1+\alpha}}{\left(h / b_{\alpha}\right)^{\beta}-1+\frac{\beta}{1+\alpha}} & ; & b_{\alpha}<x<h \\
0 & ; & h \leq x<\infty
\end{array}\right\} .
$$

Then the pair $\left(b_{\alpha}, g_{\alpha}(\cdot)\right)$ solves the Variational Inequality 4.1 .

Proof : The general solution of the equation (4.4) is given by $g_{\alpha}(x)=A_{\alpha} x^{-\gamma_{+}}+B_{\alpha} x^{-\gamma_{-}}$ for suitable real constants $A_{\alpha}, B_{\alpha}$, and $\gamma_{ \pm}$as in the proof of Proposition 2.1. The requirements $g_{\alpha}\left(b_{\alpha}+\right)=q-b_{\alpha}, g_{\alpha}^{\prime}\left(b_{\alpha}+\right)=-1$ (continuity of the function $g_{\alpha}(\cdot)$ and of its derivative at $\left.x=b_{\alpha}\right)$ and (4.9), lead to the equation (4.14) for $b_{\alpha}$ and to the expressions

$$
A_{\alpha}=\frac{\left(\frac{q-b_{\alpha}}{b_{\alpha}}\right) h^{\beta}}{\left(\frac{h}{b_{\alpha}}\right)^{\beta}-\left(1-\frac{\beta}{1+\alpha}\right)}, \quad B_{\alpha}=-A_{\alpha}\left(1-\frac{\beta}{1+\alpha}\right) h^{-\beta} .
$$

These, in turn, yield the expression of (4.15) for $g_{\alpha}(\cdot)$.

To show that the equation (4.14) has a unique solution in the interval $\left(0, \frac{\alpha q}{1+\alpha}\right)$, write it in the form $F_{\alpha}\left(b_{\alpha}\right)=0$, where $F_{\alpha}(\cdot)$ is the strictly convex function

$$
F_{\alpha}(u) \triangleq \beta+\left(1-\frac{\beta}{1+\alpha}\right)\left(\frac{u}{h}\right)^{\beta}-1-\beta\left(\frac{u}{q}\right)=F(u)-\frac{\beta}{1+\alpha}\left(\frac{u}{h}\right)^{\beta}, \quad 0 \leq u<\infty
$$

(recall here that $1+\alpha>\beta>1$ ). We have $F_{\alpha}(0)=\beta-1>0$ as well as

$$
F_{\alpha}\left(\frac{\alpha q}{1+\alpha}\right)=\beta-1+\left(1-\frac{\beta}{1+\alpha}\right)\left(\frac{\alpha}{1+\alpha} \frac{q}{h}\right)^{\beta}-\frac{\alpha \beta}{1+\alpha}<\beta-1+\left(1-\frac{\beta}{1+\alpha}\right)-\frac{\alpha \beta}{1+\alpha}=0 .
$$

Consequently, there is a unique root $b_{\alpha}$ of the equation $F_{\alpha}(u)$ in the interval $\left(0, \frac{\alpha q}{1+\alpha}\right)$. This root satisfies $F\left(b_{\alpha}\right)=\frac{\beta}{1+\alpha}\left(\frac{b}{h}\right)^{\beta}>0$, so we have also $b_{\alpha}<b$.

The derivatives of the function $g_{\alpha}(\cdot)$ in $(4.15)$ are given as

$$
g_{\alpha}^{\prime}(x)=\left\{\begin{array}{cll}
-1 & ; & 0<x<b_{\alpha} \\
B_{\alpha}-A_{\alpha} \gamma_{+} x^{-\beta} & ; & b_{\alpha}<x<h \\
0 & ; & x>h
\end{array}\right\}
$$

and

$$
g_{\alpha}^{\prime \prime}(x)=\left\{\begin{array}{cl}
A_{\alpha} \beta \gamma_{+} x^{-(1+\beta)} & ; \quad b_{\alpha}<x<h \\
0 & ; \quad 0<x<b_{\alpha} \text { or } x>h
\end{array}\right\} .
$$

Thus, in order to verify the decrease and convexity of $g_{\alpha}(\cdot)$, it is enough to check that $B_{\alpha}<0<A_{\alpha}$; but this follows readily from the expressions of (4.16) under our assumptions.

To verify (4.5) and (4.8), note that we have

$$
\alpha g_{\alpha}(x)+x g_{\alpha}^{\prime}(x)=\alpha(q-x)-x>\alpha q-(1+\alpha) b_{\alpha}>0, \quad \text { on }\left(0, b_{\alpha}\right)
$$




$$
\frac{\sigma^{2}}{2} x^{2} g_{\alpha}^{\prime \prime}(x)+r x g_{\alpha}^{\prime}(x)-r g_{\alpha}(x)=-r x-r(q-x)=-r q<0, \quad \text { on }\left(0, b_{\alpha}\right)
$$

as well as

$$
\begin{aligned}
\alpha g_{\alpha}(x)+x g_{\alpha}^{\prime}(x) & =\alpha\left(A_{\alpha} x^{-\gamma_{+}}+B_{\alpha} x\right)+x\left(-A_{\alpha} \gamma_{+} x^{-\beta}+B_{\alpha}\right) \\
& =A_{\alpha} x\left(\alpha-\gamma_{+}\right)\left(x^{-\beta}-h^{-\beta}\right)>0, \quad \text { on }\left(b_{\alpha}, h\right) .
\end{aligned}
$$

The equality (4.7) is clear from (4.3) and $b_{\alpha}<\frac{\alpha q}{1+\alpha}$, so it remains to show (4.6), namely

$$
\begin{array}{r}
g_{\alpha}(x)>q-x \quad ; \quad b_{\alpha}<x \leq \frac{\alpha q}{1+\alpha} \\
x^{\alpha} g_{\alpha}(x)>\frac{q}{1+\alpha}\left(\frac{\alpha q}{1+\alpha}\right)^{\alpha} \quad ; \quad \frac{\alpha q}{1+\alpha}<x<h .
\end{array}
$$

The inequality (4.17) follows easily, from the strict convexity of $g_{\alpha}(\cdot)$ on the interval $\left(b_{\alpha}, h\right)$ and from $g_{\alpha}^{\prime}\left(b_{\alpha}+\right)=-1, g_{\alpha}\left(b_{\alpha}+\right)=q-b_{\alpha}$. To see (4.18), notice that

$$
\text { the function } \quad x \mapsto x^{\alpha} g_{\alpha}(x) \text { is strictly increasing on }\left(\frac{\alpha q}{1+\alpha}, h\right)
$$

since $\left(x^{\alpha} g_{\alpha}(x)\right)^{\prime}=x^{\alpha-1}\left(\alpha g_{\alpha}(x)+x g_{\alpha}^{\prime}(x)\right)>0$ from (4.8); consequently,

$$
x^{\alpha} g_{\alpha}(x)>\left(\frac{\alpha q}{1+\alpha}\right)^{\alpha} g_{\alpha}\left(\frac{\alpha q}{1+\alpha}\right) \geq\left(\frac{\alpha q}{1+\alpha}\right)^{\alpha}\left(q-\frac{\alpha q}{1+\alpha}\right)=\frac{q}{1+\alpha}\left(\frac{\alpha q}{1+\alpha}\right)^{\alpha} .
$$

4.4 REMARK : In terms of the process $\Lambda^{*}(\cdot) \in \mathcal{L}^{+}$of $(3.14)$, the optimal hedging portfolio $\hat{\pi}_{\alpha}(\cdot)$ and the optimal cumulative consumption processes $\hat{C}_{\alpha}(\cdot)$ are given by

$$
\begin{gathered}
\hat{\pi}_{\alpha}(t)=\left\{\begin{array}{cc}
\left.e^{-\alpha \Lambda^{*}(t)}\left(x g_{\alpha}^{\prime}(x)\right)\right|_{x=P_{\Lambda^{*}}(t)} & ; \quad 0 \leq t<\tau_{h}^{*} \\
0 & ; \quad \tau_{h}^{*} \leq t<\infty
\end{array}\right\}, \\
\hat{C}_{\alpha}(t)=r q \int_{0}^{t \wedge \tau_{h}^{*}} e^{-\alpha \Lambda^{*}(s)} 1_{\left(0, b_{\alpha}\right)}\left(P_{\Lambda^{*}}(s)\right) d s
\end{gathered}
$$

respectively. The wealth process

$$
\hat{X}_{\alpha}(t) \equiv X^{g_{\alpha}(x), \hat{\pi}_{\alpha}, \hat{C}_{\alpha}}(t)=\left\{\begin{array}{cc}
e^{-\alpha \Lambda^{*}(t)} g_{\alpha}\left(P_{\Lambda^{*}}(t)\right) & ; \quad 0 \leq t<\tau_{h}^{*} \\
0 & ; \quad \tau_{h}^{*} \leq t<\infty
\end{array}\right\}
$$

is then the value process for the American put-option of barrier type, under the short-selling constraint of (3.2). Note that this constraint is indeed satisfied by the portfolio-proportion process

$$
\hat{p}_{\alpha}(t) \triangleq\left\{\begin{array}{cc}
\hat{\pi}_{\alpha}(t) / \hat{X}_{\alpha}(t)=\left.\left(\frac{x g_{\alpha}^{\prime}(x)}{g_{\alpha}(x)}\right)\right|_{x=P_{\Lambda^{*}}(t)} & ; \quad 0 \leq t<\tau_{h}^{*} \\
0 & ; \quad \tau_{h}^{*} \leq t<\infty
\end{array}\right\},
$$

for which we clearly have $\hat{p}_{\alpha}(\cdot) \geq-\alpha$.

Finally, the stopping time $\tau^{*} \triangleq \inf \left\{t \geq 0 / \hat{C}_{\alpha}(t)>0\right\} \equiv \tau_{b_{\alpha}}^{*}$ of (4.11) is the optimal exercise time of the option by its holder. 


\section{THE CASE $0<\alpha \leq 2 r / \sigma^{2}$}

Now let us return to the case $\alpha \leq 2 r / \sigma^{2}$ of "severely constrained" short-selling of stock. In this setting the hedging problem admits a very simple solution, given by the function $\phi_{\alpha}(\cdot)$ of $(4.3)$.

5.1 THEOREM: Suppose that $0<\alpha \leq 2 r / \sigma^{2}$. Then the hedging price of (3.4) is given as

$$
G_{\alpha}(x)=\left\{\begin{array}{cc}
\phi_{\alpha}(x) & ; \quad 0<x<h \\
0 & ; \quad h \leq x<\infty
\end{array}\right\}
$$

where $\phi_{\alpha}(\cdot)$ is the $\alpha$-enlargement of the function $\phi(\cdot)=(q-\cdot)^{+}$, as in $(4.3)$.

We shall devote much of the remainder of this section to the proof of Theorem 5.1 for $x \in$ $(0, h)$. Let us start by noticing that the function $\phi_{\alpha}(\cdot)$ of $(4.3)$ is convex, decreasing and of class $\mathcal{C}([0, \infty)) \cap \mathcal{C}_{b}^{1}((0, \infty)) \cap \mathcal{C}_{b}^{2}\left((0, \infty) \backslash\left\{\frac{\alpha q}{1+\alpha}\right\}\right)$, with

$$
\phi_{\alpha}^{\prime}(x)=\left\{\begin{array}{cl}
-1 & ; \quad 0<x<\frac{\alpha q}{1+\alpha} \\
-\left(\frac{\alpha q}{1+\alpha}\right)^{1+\alpha} x^{-(1+\alpha)} & ; \quad x \geq \frac{\alpha q}{1+\alpha}
\end{array}\right\}
$$

and

$$
\phi_{\alpha}^{\prime \prime}(x)=\left\{\begin{array}{cl}
0 & ; \quad 0<x<\frac{\alpha q}{1+\alpha} \\
\alpha q\left(\frac{\alpha q}{1+\alpha}\right)^{\alpha} x^{-(2+\alpha)} & ; \quad x>\frac{\alpha q}{1+\alpha}
\end{array}\right\} .
$$

In particular, we have

$$
\begin{gathered}
\alpha \phi_{\alpha}(x)+x \phi_{\alpha}^{\prime}(x)=\left\{\begin{array}{cc}
\alpha q-x(1+\alpha)>0 & ; 0<x<\frac{\alpha q}{1+\alpha} \\
0 \quad & ; \quad x \geq \frac{\alpha q}{1+\alpha}
\end{array}\right\} \\
\frac{\sigma^{2}}{2} x^{2} \phi_{\alpha}^{\prime \prime}(x)+r x \phi_{\alpha}^{\prime}(x)-r \phi_{\alpha}(x)=\left\{\begin{array}{cl}
-r q \quad & 0<x<\frac{\alpha q}{1+\alpha} \\
\left(\frac{\alpha \sigma^{2}}{2 r}-1\right) r q x^{-\alpha}\left(\frac{\alpha q}{1+\alpha}\right)^{\alpha} \leq 0 & ; \quad x>\frac{\alpha q}{1+\alpha}
\end{array}\right\} .
\end{gathered}
$$

For fixed $0<x<h$, and an arbitrary $\Lambda(\cdot) \in \mathcal{L}^{+}$, let us apply Itô's rule (e.g. Karatzas \& Shreve (1991), Problem 3.7.3, p.219) to the process $e^{-r t-\alpha \Lambda(t)} \phi_{\alpha}\left(P_{\Lambda}(t)\right), 0 \leq t<\infty$. By analogy with (6.1)-(6.3) in the Appendix A, and using again the inequalities of (5.2) and (5.3) in conjuction with the dynamics of $(3.13)$ for the process $P_{\Lambda}(\cdot)$, we obtain

$$
\phi_{\alpha}(x) \geq \mathbf{E}_{0}\left[e^{-r \tau-\alpha \Lambda(\tau)} \phi_{\alpha}\left(P_{\Lambda}(\tau)\right) 1_{\left\{\tau<\tau_{h}^{\Lambda}\right\}}\right] \geq \mathbf{E}_{0}\left[e^{-r \tau-\alpha \Lambda(\tau)}\left(q-P_{\Lambda}(\tau)\right)^{+} 1_{\left\{\tau<\tau_{h}^{\Lambda}\right\}}\right], \quad \forall \tau \in \mathcal{S} .
$$

Therefore,

$$
\begin{aligned}
\phi_{\alpha}(x) & \geq \sup _{\Lambda(\cdot) \in \mathcal{L}^{+}} \sup _{\tau \in \mathcal{S}} \mathbf{E}_{0}\left[e^{-r \tau-\alpha \Lambda(\tau)} \phi_{\alpha}\left(P_{\Lambda}(\tau)\right) 1_{\left\{\tau<\tau_{h}^{\Lambda}\right\}}\right] \\
& \geq \sup _{\Lambda(\cdot) \in \mathcal{L}^{+}} \sup _{\tau \in \mathcal{S}} \mathbf{E}_{0}\left[e^{-r \tau-\alpha \Lambda(\tau)}\left(q-P_{\Lambda}(\tau)\right)^{+} 1_{\left\{\tau<\tau_{h}^{\Lambda}\right\}}\right] \\
& \geq \sup _{\lambda(\cdot) \in \mathcal{D}} \sup _{\tau \in \mathcal{S}} \mathbf{E}_{0}\left[e^{-r \tau-\alpha \Lambda(\tau)}\left(q-P_{\Lambda}(\tau)\right)^{+} 1_{\left\{\tau<\tau_{h}^{\Lambda}\right\}}\right] .
\end{aligned}
$$




\subsection{LEMMA: The inequalities of (5.4) are in fact valid as equalities.}

Proof: Clearly, it suffices to verify

$$
\sup _{\lambda(\cdot) \in \mathcal{D}} \mathbf{E}_{0}\left[e^{-r T-\alpha \Lambda(T)}\left(q-P_{\Lambda}(T)\right)^{+} 1_{\left\{T<\tau_{h}^{\Lambda}\right\}}\right] \stackrel{T \downarrow 0}{\longrightarrow} \phi_{\alpha}(x),
$$

where the expression on the left-hand side is the value of the European-type barrier option with fixed exercise time $T>0$. From Wystup (1997), we know that this value equals

$$
\sup _{\Lambda(\cdot) \in \mathcal{L}_{r}^{+}(T)} \mathbf{E}_{0}\left[e^{-r T-\alpha \Lambda(T)}\left(q-P_{\Lambda}(T)\right)^{+} 1_{\left\{T<\tau_{h}^{\Lambda}\right\}}\right]=\mathbf{E}_{0}\left[e^{-r T-\alpha \Lambda_{T}^{*}(T)}\left(q-P_{\Lambda_{T}^{*}}(T)\right)^{+} 1_{\left\{T<\tau_{h}^{*}\right\}}\right],
$$

where we have set

$$
\Lambda_{T}^{*}(t)=\left\{\begin{array}{cl}
\max _{0 \leq u \leq t}\left(\log \left(\frac{S(u)}{h}\right)\right)^{+} & ; \quad 0 \leq t<T \\
\max \left[\Lambda_{T}^{*}(T-), \log \left(\frac{1+\alpha}{\alpha q} S(T)\right)\right] & ; \quad t=T
\end{array}\right\} .
$$

In other words, $\Lambda_{T}^{*}(\cdot)$ pushes the stock-price just enough to avoid crossing the barrier $h$, and makes a (possible) jump at $t=T$ that pushes $P_{\Lambda_{T}^{*}}(T)$ to $\left(\frac{\alpha q}{1+\alpha}\right)$ whenever the stock-price $S(T)$ at time $t=T$ exceeds this threshold. Accordingly, we have denoted in (5.6) by $\mathcal{L}_{r}^{+}(T)$ the space of adapted processes $\Lambda:[0, T] \times \Omega \longrightarrow[0, \infty)$ whose paths are increasing and right-continuous on $[0, T)$ with $\Lambda(0)=0$. With this notation, we have clearly

$$
\begin{aligned}
\Lambda_{T}^{*}(T) & \stackrel{T \downarrow 0}{\longrightarrow}\left(\log x-\log \left(\frac{\alpha q}{1+\alpha}\right)\right)^{+}, \\
P_{\Lambda_{T}^{*}}(T) & \stackrel{T \downarrow 0}{\longrightarrow} \min \left(x, \frac{\alpha q}{1+\alpha}\right), \\
1_{\left\{T<\tau_{h}^{*}\right\}} & \stackrel{T \downarrow 0}{\longrightarrow} 1, \text { a.s. }
\end{aligned}
$$

By the Bounded Convergence Theorem, the limit of the left-hand side in (5.6) as $T \downarrow 0$, is equal to

$$
\exp \left[-\alpha\left\{\log x-\log \left(\frac{\alpha q}{1+\alpha}\right)\right\}^{+}\right]\left(q-\left(x \wedge \frac{\alpha q}{1+\alpha}\right)\right)=\phi_{\alpha}(x)
$$

as claimed.

Now fix $0 \leq x<h$ and, for arbitrary $\lambda(\cdot) \in \mathcal{D}$, apply Itô's rule to the process $e^{-r t-\alpha \Lambda(t)} \phi_{\alpha}(S(t))$, $0 \leq t<\infty$. By analogy with (6.5)-(6.6) in Appendix A, and using the inequalities of (5.2) and (5.3), we obtain in conjunction with the dynamics of (3.8) for the stock-price process $S(\cdot)$ :

$$
\phi_{\alpha}(x) \geq \mathbf{E}_{\lambda}\left[e^{-r \tau-\alpha \Lambda(\tau)} \phi_{\alpha}(S(\tau)) 1_{\left\{\tau<\tau_{h}\right\}}\right] \geq \mathbf{E}_{\lambda}\left[e^{-r \tau-\alpha \Lambda(\tau)}(q-S(\tau))^{+} 1_{\left\{\tau<\tau_{h}\right\}}\right], \quad \forall \tau \in \mathcal{S} .
$$

Consequently,

$$
\begin{aligned}
\phi_{\alpha}(x) & \geq \sup _{\lambda(\cdot) \in \mathcal{D}} \sup _{\tau \in \mathcal{S}} \mathbf{E}_{\lambda}\left[e^{-r \tau-\alpha \Lambda(\tau)} \phi_{\alpha}(S(\tau)) 1_{\left\{\tau<\tau_{h}\right\}}\right] \\
& \geq \sup _{\lambda(\cdot) \in \mathcal{D}} \sup _{\tau \in \mathcal{S}} \mathbf{E}_{\lambda}\left[e^{-r \tau-\alpha \Lambda(\tau)}(q-S(\tau))^{+} 1_{\left\{\tau<\tau_{h}\right\}}\right]=: G_{\alpha}(x) .
\end{aligned}
$$


5.3 Proposition: The inequalities of (5.8) can be replaced by equalities.

Proof: It suffices to show the inequality $\phi_{\alpha}(x) \leq G_{\alpha}(x)$; but this is proved in exactly the same way as in the Appendix A (proof of (6.7)). This completes the proof of Theorem 5.1.

5.4 REMARK : By analogy with Remark 4.3, the optimal hedging pair $\left(\hat{\pi}_{\alpha}(\cdot), \hat{C}_{\alpha}(\cdot)\right)$ is now given as

$$
\hat{\pi}_{\alpha}(t)=\left\{\begin{array}{cc}
\left.e^{-\alpha \Lambda^{*}(t)}\left(x \phi_{\alpha}^{\prime}(x)\right)\right|_{x=P_{\Lambda^{*}}(t)} & ; \quad 0 \leq t<\tau_{h}^{*} \\
0 & ; \quad \tau_{h}^{*} \leq t<\infty
\end{array}\right\}
$$

and

$$
\begin{aligned}
\hat{C}_{\alpha}(t)=r q \int_{0}^{t \wedge \tau_{h}^{*}} e^{-\alpha \Lambda^{*}(u)}\left[1_{\left(0, \frac{\alpha q}{1+\alpha}\right)}\left(P_{\Lambda^{*}}(u)\right)\right. \\
\left.\quad+\left(1-\frac{\alpha \sigma^{2}}{2 r}\right)\left(\frac{\frac{\alpha q}{1+\alpha}}{P_{\Lambda^{*}}(u)}\right)^{\alpha} 1_{\left[\frac{\alpha q}{1+\alpha}, h\right)}\left(P_{\Lambda^{*}}(u)\right)\right] d u
\end{aligned}
$$

for $0 \leq t<\infty$, whereas the corresponding value-process is

$$
\hat{X}_{\alpha}(t) \equiv X^{\phi_{\alpha}(x), \hat{\pi}_{\alpha}, \hat{C}_{\alpha}}(t)=\left\{\begin{array}{cc}
e^{-\alpha \Lambda^{*}(t)} \phi_{\alpha}\left(P_{\Lambda^{*}}(t)\right) & ; \quad 0 \leq t<\tau_{h}^{*} \\
0 & ; \quad \tau_{h}^{*} \leq t<\infty
\end{array}\right\} .
$$

The associated portfolio-proportion process

$$
\hat{p}_{\alpha}(t) \triangleq\left\{\begin{array}{cc}
\hat{\pi}_{\alpha}(t) / \hat{X}_{\alpha}(t)=\left.\left(\frac{x \phi_{\alpha}^{\prime}(x)}{\phi_{\alpha}(x)}\right)\right|_{x=P_{\Lambda^{*}}(t)} & ; \quad 0 \leq t<\tau_{h}^{*} \\
0 & ; \quad \tau_{h}^{*} \leq t<\infty
\end{array}\right\}
$$

clearly satisfies the constraint $\hat{p}_{\alpha}(\cdot) \geq-\alpha$.

The optimal time for the holder to exercise his option, is

$$
\hat{\tau}_{\alpha} \triangleq \inf \left\{t \geq 0 / \hat{C}_{\alpha}(t)>0\right\}
$$

This coincides with the stopping time $\tau^{*} \triangleq \inf \left\{t \geq 0 / P_{\Lambda^{*}}(t) \leq \alpha q /(1+\alpha)\right\}$ if $\alpha \equiv 2 r / \sigma^{2}$; but for $\alpha<2 r / \sigma^{2}$, we have $\hat{\tau}_{\alpha} \equiv 0$ in (5.13). Both these claims follow readily from (5.10).

5.5 REMARK: The limiting case $\alpha \downarrow 0$ corresponds to prohibition of short-selling in (3.2). In this limiting case, the cheapest way for the issuer to hedge the American barrier option of (2.2) is the trivial one of setting aside the strike-price itself at $t=0$, namely

$$
\lim _{\alpha \downarrow 0} H_{\alpha}(x)=\lim _{\alpha \downarrow 0} G_{\alpha}(x)=\left\{\begin{aligned}
\lim _{\alpha \downarrow 0} \phi_{\alpha}(x) & ; \quad 0<x<h \\
0 & ; \quad h \leq x<\infty
\end{aligned}\right\}=\left\{\begin{array}{ll}
q & ; \quad 0<x<h \\
0 & ; \quad h \leq x<\infty
\end{array}\right\}
$$

as can be checked easily from (5.1), (4.3). 


\section{APPENDIX A}

We devote this section to the proof of Theorem 4.2. All the terms in (4.12) are equal to zero for $x \geq h$, so we shall concentrate on the case $0<x<h$. On the interval $(0, h)$, the functions $g_{\alpha}(\cdot), g_{\alpha}^{\prime}(\cdot)$ are bounded, continuous; and the function $g_{\alpha}^{\prime \prime}(\cdot)$ is bounded, continuous on $(0, h) \backslash\left\{b_{\alpha}\right\}$.

For any given $\Lambda(\cdot) \in \mathcal{L}^{+}$and $\tau \in \mathcal{S}$, apply Itô's (e.g. Karatzas \& Shreve (1991), Problem 3.7.3, p. 219) rule to the process $e^{-r t-\alpha \Lambda(t)} g_{\alpha}\left(P_{\Lambda}(t)\right), 0 \leq t<\tau_{h}^{\Lambda}$ in conjunction with (3.13), to obtain

$$
\begin{aligned}
& g_{\alpha}(x)-e^{-r\left(\tau \wedge \tau_{h}^{\Lambda}\right)-\alpha \Lambda\left(\tau \wedge \tau_{h}^{\Lambda}\right)} g_{\alpha}\left(P_{\Lambda}\left(\tau \wedge \tau_{h}^{\Lambda}\right)\right)+\left.\sigma \int_{0}^{\tau \wedge \tau_{h}^{\Lambda}} e^{-r t-\alpha \Lambda(t)}\left(\xi g_{\alpha}^{\prime}(\xi)\right)\right|_{\xi=P_{\Lambda}(t)} d W_{0}(t) \\
= & \left.\int_{0}^{\tau \wedge \tau_{h}^{\Lambda}} e^{-r t-\alpha \Lambda(t)}\left(\xi g_{\alpha}^{\prime}(\xi)+\alpha g_{\alpha}(\xi)\right)\right|_{\xi=P_{\Lambda}(t)} d \Lambda(t) \\
& -\left.\int_{0}^{\tau \wedge \tau_{h}^{\Lambda}} e^{-r t-\alpha \Lambda(t)}\left(\frac{\sigma^{2}}{2} \xi^{2} g_{\alpha}^{\prime \prime}(\xi)+r \xi g_{\alpha}^{\prime}(\xi)-r g_{\alpha}(\xi)\right)\right|_{\xi=P_{\Lambda}(t)} d t \geq 0, \quad \mathbf{P}_{0} \text {-a.s. }
\end{aligned}
$$

from (4.4), (4.5) and (4.8), (4.9). Note also that, thanks to (4.4),(4.9) and (3.15), the inequality of (6.1) holds as equality for

$$
\Lambda(\cdot) \equiv \Lambda^{*}(\cdot), \quad \tau \equiv \tau_{b_{\alpha}}^{*}
$$

defined in (3.14) and (4.11), respectively. The same argument as in the proof of Theorem 2.3 shows that the stochastic integral in (6.1) has $\mathbf{P}_{0}$-expectation equal to zero. We deduce

$$
\begin{aligned}
g_{\alpha}(x) & \geq \mathbf{E}_{0}\left[e^{-r\left(\tau \wedge \tau_{h}^{\Lambda}\right)-\alpha \Lambda\left(\tau \wedge \tau_{h}^{\Lambda}\right)} g_{\alpha}\left(P_{\Lambda}\left(\tau \wedge \tau_{h}^{\Lambda}\right)\right)\right] \\
& \geq \mathbf{E}_{0}\left[e^{-r \tau-\alpha \Lambda(\tau)} g_{\alpha}\left(P_{\Lambda}(\tau)\right) 1_{\left\{\tau<\tau_{h}^{\Lambda}\right\}}\right] \\
& \geq \mathbf{E}_{0}\left[e^{-r \tau-\alpha \Lambda(\tau)} \phi_{\alpha}\left(P_{\Lambda}(\tau)\right) 1_{\left\{\tau<\tau_{h}^{\Lambda}\right\}}\right] \\
& \geq \mathbf{E}_{0}\left[e^{-r \tau-\alpha \Lambda(\tau)}\left(q-P_{\Lambda}(\tau)\right)^{+} 1_{\left\{\tau<\tau_{h}^{\Lambda}\right\}}\right]
\end{aligned}
$$

and note that the inequalities of (6.3) hold as equalities for the choices of (6.2). Therefore,

$$
\begin{aligned}
g_{\alpha}(x) & =\mathbf{E}_{0}\left[e^{-r \tau_{b_{\alpha}}^{*}-\alpha \Lambda^{*}\left(\tau_{b_{\alpha}}^{*}\right)}\left(q-P_{\Lambda^{*}}\left(\tau_{b_{\alpha}}^{*}\right)\right)^{+} 1_{\left\{\tau_{b_{\alpha}}^{*}<\tau_{h}^{*}\right\}}\right] \\
& =\sup _{\tau \in \mathcal{S}} \mathbf{E}_{0}\left[e^{-r \tau-\alpha \Lambda^{*}(\tau)}\left(q-P_{\Lambda^{*}}(\tau)\right)^{+} 1_{\left\{\tau<\tau_{h}^{*}\right\}}\right] \\
& =\sup _{\Lambda(\cdot) \in \mathcal{L}^{+}} \sup _{\tau \in \mathcal{S}} \mathbf{E}_{0}\left[e^{-r \tau-\alpha \Lambda(\tau)}\left(q-P_{\Lambda}(\tau)\right)^{+} 1_{\left\{\tau<\tau_{h}^{\Lambda}\right\}}\right] \\
& =\sup _{\lambda(\cdot) \in \mathcal{D}} \sup _{\tau \in \mathcal{S}} \mathbf{E}_{0}\left[e^{-r \tau-\alpha \Lambda(\tau)}\left(q-P_{\Lambda}(\tau)\right)^{+} 1_{\left\{\tau<\tau_{h}^{\Lambda}\right\}}\right],
\end{aligned}
$$

where the last equality comes from Appendix B. Similar equalities hold if one replaces the function $(q-\cdot)^{+}=\phi(\cdot)$ in the equalities of $(6.4)$, by its $\alpha$-enlargement $\phi_{\alpha}(\cdot)$ of (4.3). This proves all the equalities of (4.12) except the first, namely, $G_{\alpha}(x)=g_{\alpha}(x)$.

Proof of $G_{\alpha}(x) \leq g_{\alpha}(x)$ : For any given $\lambda(\cdot) \in \mathcal{D}$ and $\tau \in \mathcal{S}$, and with $\Lambda(\cdot) \equiv \int_{0}^{\cdot} \lambda(u) d u$, 
apply Itô's rule to the process $e^{-r t-\alpha \Lambda(t)} g_{\alpha}(S(t)), 0 \leq t<\tau_{h}$ in conjunction with (3.8) and (2.3), to obtain

$$
\begin{aligned}
& g_{\alpha}(x)-e^{-r\left(\tau \wedge \tau_{h}\right)-\alpha \Lambda\left(\tau \wedge \tau_{h}\right)} g_{\alpha}\left(S\left(\tau \wedge \tau_{h}\right)\right)+\left.\sigma \int_{0}^{\tau \wedge \tau_{h}} e^{-r t-\alpha \Lambda(t)}\left(\xi g_{\alpha}^{\prime}(\xi)\right)\right|_{\xi=S(t)} d W_{\lambda}(t) \\
= & \left.\int_{0}^{\tau \wedge \tau_{h}} e^{-r t-\alpha \Lambda(t)}\left(\xi g_{\alpha}^{\prime}(\xi)+\alpha g_{\alpha}(\xi)\right)\right|_{\xi=S(t)} \lambda(t) d t \\
& -\left.\int_{0}^{\tau \wedge \tau_{h}} e^{-r t-\alpha \Lambda(t)}\left(\frac{\sigma^{2}}{2} \xi^{2} g_{\alpha}^{\prime \prime}(\xi)+r \xi g_{\alpha}^{\prime}(\xi)-r g_{\alpha}(\xi)\right)\right|_{\xi=S(t)} d t \quad \geq 0, \quad \mathbf{P}_{\lambda}-\text { a.s. }
\end{aligned}
$$

thanks to (4.4) - (4.9), by analogy with (6.1). Just as before (cf. proof of Theorem 2.3), the $\mathbf{P}_{\lambda}$-expectation of the stochastic integral in (6.5) is equal to zero, which gives

$$
\begin{aligned}
g_{\alpha}(x) & \geq \mathbf{E}_{\lambda}\left[e^{-r\left(\tau \wedge \tau_{h}\right)-\alpha \Lambda\left(\tau \wedge \tau_{h}\right)} g_{\alpha}\left(S\left(\tau \wedge \tau_{h}\right)\right)\right] \geq \mathbf{E}_{\lambda}\left[e^{-r \tau-\alpha \Lambda(\tau)} g_{\alpha}(S(\tau)) 1_{\left\{\tau<\tau_{h}\right\}}\right] \\
& \geq \mathbf{E}_{\lambda}\left[e^{-r \tau-\alpha \Lambda(\tau)}(q-S(\tau))^{+} 1_{\left\{\tau<\tau_{h}\right\}}\right]
\end{aligned}
$$

by analogy with (6.3). Taking the supremum in (6.6) with respect to $\lambda(\cdot) \in \mathcal{D}, \tau \in \mathcal{S}$ and recalling (3.4), (3.5), we obtain the inequality $g_{\alpha}(x) \geq G_{\alpha}(x)$.

Proof of $G_{\alpha}(x) \geq g_{\alpha}(x)$ : In view of (3.4),(3.3) and (6.4), it suffices to show that

$$
\xi \geq \mathbf{E}_{0}\left[e^{-r \tau-\alpha \Lambda(\tau)}\left(q-P_{\Lambda}(\tau)\right)^{+} 1_{\left\{\tau<\tau_{h}^{\Lambda}\right\}}\right], \quad \forall \lambda(\cdot) \in \mathcal{D}, \tau \in \mathcal{S}
$$

holds for all $\xi>H_{\alpha}(x)$. We can rewrite the right-hand side of (6.7) as

$$
\begin{aligned}
& \tilde{\mathbf{E}}_{\lambda}\left[\left.\frac{d \mathbf{P}_{0}}{d \tilde{\mathbf{P}}_{\lambda}}\right|_{\mathcal{F}(\tau)} \cdot e^{-r \tau-\alpha \Lambda(\tau)}\left(q-P_{\Lambda}(\tau)\right)^{+} 1_{\left\{\tau<\tau_{h}^{\Lambda}\right\}}\right] \\
= & \tilde{\mathbf{E}}_{\lambda}\left[\left(\tilde{Z}_{\lambda}(\tau)\right)^{-1} \cdot e^{-r \tau-\alpha \Lambda(\tau)}\left(q-P_{\Lambda}(\tau)\right)^{+} 1_{\left\{\tau<\tau_{h}^{\Lambda}\right\}}\right],
\end{aligned}
$$

where $\tilde{\mathbf{E}}_{\lambda}$ denotes expectation with respect to the probability measure $\tilde{\mathbf{P}}_{\lambda}$ that satisfies

$$
\left.\frac{d \tilde{\mathbf{P}}_{\lambda}}{d \mathbf{P}_{0}}\right|_{\mathcal{F}(t)}=\tilde{Z}_{\lambda}(t) \triangleq \exp \left\{\int_{0}^{t}\left(\frac{\lambda(s)}{\sigma}\right) d W_{0}(s)-\frac{1}{2} \int_{0}^{t}\left(\frac{\lambda(s)}{\sigma}\right)^{2} d s\right\}
$$

for all $0 \leq t<\infty$.

6.1 REMARK: Under the measure $\tilde{\mathbf{P}}_{\lambda}$, the process

$$
\tilde{W}_{\lambda}(t) \triangleq W_{0}(t)-\int_{0}^{t} \frac{\lambda(u)}{\sigma} d u, \quad 0 \leq t<\infty
$$

is Brownian motion, and thus

$$
d P_{\Lambda}(t)=P_{\Lambda}(t)\left[r d t+\sigma d \tilde{W}_{\lambda}(t)\right]
$$


from (3.13). In other words, the process $P_{\Lambda}(\cdot)$ has the same law under $\tilde{\mathbf{P}}_{\lambda}$, as the law of process $S(\cdot)$ under $\mathbf{P}_{0}$. Furthermore, we have the dynamics

$$
d\left(\tilde{Z}_{\lambda}(t)\right)^{-1}=-\left(\frac{\lambda(t)}{\sigma}\right)\left(\tilde{Z}_{\lambda}(t)\right)^{-1} d \tilde{W}_{\lambda}(t), \quad\left(\tilde{Z}_{\lambda}(0)\right)^{-1} \equiv 1
$$

for the inverse of the process $\tilde{Z}_{\lambda}(\cdot)$ in $(6.9)$.

From the assumption $\xi>H_{\alpha}(x)$, we know that there exists a portfolio / consumption process pair $(\tilde{\pi}, \tilde{C})$ such that, for the corresponding wealth process $\tilde{X}_{\lambda}(\cdot) \equiv X^{\xi, \tilde{\pi}, \tilde{C}}(\cdot)$ as in

$$
e^{-r t} \tilde{X}_{\lambda}(t)=\xi-\int_{(0, t]} e^{-r s} d \tilde{C}(s)+\sigma \int_{0}^{t} e^{-r u} \tilde{\pi}(u) d \tilde{W}_{\lambda}(u)
$$

(by analogy with (2.5)), we have both

$$
\alpha \tilde{X}_{\lambda}(t)+\tilde{\pi}(t) \geq 0, \quad 0 \leq t<\infty
$$

and

$$
\tilde{X}_{\lambda}(t) \geq\left(q-P_{\Lambda}(t)\right)^{+} 1_{\left\{t<\tau_{h}^{\Lambda}\right\}}, \quad 0 \leq t<\infty
$$

(by analogy with (3.2) and (2.4), (2.2)). Now in order to prove (6.7), it suffices to show that the process

$$
\tilde{Q}_{\lambda}(t) \triangleq\left(\tilde{Z}_{\lambda}(t)\right)^{-1} \cdot e^{-r t-\alpha \Lambda(t)} \tilde{X}_{\lambda}(t), \quad 0 \leq t<\infty
$$

is a $\tilde{\mathbf{P}}_{\lambda}$-supermartingale; because then from the optional sampling theorem and (6.15), we have

$$
\begin{aligned}
\xi=\tilde{Q}_{\lambda}(0) & \geq \tilde{\mathbf{E}}_{\lambda}\left[\tilde{Q}_{\lambda}(\tau)\right]=\tilde{\mathbf{E}}_{\lambda}\left[\left(\tilde{Z}_{\lambda}(\tau)\right)^{-1} e^{-r \tau-\alpha \Lambda(\tau)} \tilde{X}_{\lambda}(\tau)\right] \\
& \geq \tilde{\mathbf{E}}_{\lambda}\left[\left(\tilde{Z}_{\lambda}(\tau)\right)^{-1} e^{-r \tau-\alpha \Lambda(\tau)}\left(q-P_{\Lambda}(\tau)\right)^{+} 1_{\left\{\tau<\tau_{h}^{\Lambda}\right\}}\right] \\
& =\mathbf{E}_{0}\left[e^{-r \tau-\alpha \Lambda(\tau)}\left(q-P_{\Lambda}(\tau)\right)^{+} 1_{\left\{\tau<\tau_{h}^{\Lambda}\right\}}\right], \quad \forall \tau \in \mathcal{S}
\end{aligned}
$$

holds for any $\lambda(\cdot) \in \mathcal{D}$, which is $(6.7)$.

In order to prove that $\tilde{Q}_{\lambda}(\cdot)$ is a $\tilde{\mathbf{P}}_{\lambda}$-supermartingale, observe from (6.12), (6.13) that

$d \tilde{Q}_{\lambda}(t)=\left(\tilde{Z}_{\lambda}(t)\right)^{-1} e^{-r t-\alpha \Lambda(t)}\left[-\lambda(t)\left(\alpha \tilde{X}_{\lambda}(t)+\tilde{\pi}(t)\right) d t-d \tilde{C}(t)+\left(\sigma \tilde{\pi}(t)-\frac{\lambda(t)}{\sigma} \tilde{X}_{\lambda}(t)\right) d \tilde{W}_{\lambda}(t)\right]$.

Thanks to (6.14), we deduce that $\tilde{Q}_{\lambda}(\cdot)$ is a local supermartingale under $\tilde{\mathbf{P}}_{\lambda}$; but $\tilde{Q}_{\lambda}(\cdot)$ is also nonnegative, and thus it is actually a supermartingale under $\tilde{\mathbf{P}}_{\lambda}$. The proof of Theorem 4.2 is complete. 


\section{APPENDIX B}

We shall establish here the equality

$$
\begin{aligned}
& \sup _{\lambda(\cdot) \in \mathcal{D}} \sup _{\tau \in \mathcal{S}} \mathbf{E}_{0}\left[e^{-r \tau-\alpha \Lambda(\tau)}\left(q-P_{\Lambda}(\tau)\right)^{+} 1_{\left\{\tau<\tau_{h}^{\Lambda}\right\}}\right] \\
& \quad=\sup _{\Lambda(\cdot) \in \mathcal{L}^{+}} \sup _{\tau \in \mathcal{S}} \mathbf{E}_{0}\left[e^{-r \tau-\alpha \Lambda(\tau)}\left(q-P_{\Lambda}(\tau)\right)^{+} 1_{\left\{\tau<\tau_{h}^{\Lambda}\right\}}\right]
\end{aligned}
$$

which was used in the proof of Theorem 4.2 (last equality in (6.4); recall the notation of (3.12) and (4.13)). Clearly, it is enough to show that, for any given $\Lambda(\cdot) \in \mathcal{L}^{+}$and any bounded stopping time $\tau \in \mathcal{S}$, we have

$$
\begin{aligned}
L & \triangleq \sup _{\lambda(\cdot) \in \mathcal{D}} \mathbf{E}_{0}\left[e^{-r \tau-\int_{0}^{\tau} \lambda(u) d u}\left(q-e^{-\int_{0}^{\tau} \lambda(u) d u} S(\tau)\right)^{+} \cdot 1_{\left\{\max _{0 \leq u \leq \tau}\left(e^{-\int_{0}^{u} \lambda(s) d s} S(u)\right)<h\right\}}\right] \\
& \geq \mathbf{E}_{0}\left[e^{-r \tau-\alpha \Lambda(\tau)}\left(q-P_{\Lambda}(\tau)\right)^{+} 1_{\left\{\tau<\tau_{h}^{\Lambda}\right\}}\right]=: R
\end{aligned}
$$

Proceeding as in Wystup (1997), we introduce a sequence $\left\{\Lambda_{n}(\cdot)\right\}$ of "truncated left-mollifications" of $\Lambda(\cdot)$, as follows:

$$
\Lambda_{n}(t, \omega) \triangleq \int_{-1}^{0} \min \left[n, \Lambda\left(t+\frac{u}{n}, \omega\right)\right] \chi(u) d u, \quad 0 \leq t<\infty
$$

where $\chi(u)=C \cdot \exp \left\{\frac{-1}{(2 u+1)^{2}-1}\right\}$ and the constant $C>0$ is chosen so that $\int \chi(u) d u \equiv 1$. For every $\omega \in \Omega$,

(i) $\Lambda_{n}(\cdot, \omega)$ is absolutely continuous

(ii) $\Lambda_{n}(\cdot, \omega) \longrightarrow \Lambda(\cdot, \omega)$ uniformly on compact intervals as $n \longrightarrow \infty$.

Thus

$$
\begin{aligned}
L & \geq \liminf _{n} \mathbf{E}_{0}\left[e^{-r \tau-\alpha \Lambda_{n}(\tau)}\left(q-e^{-\Lambda_{n}(\tau)} S(\tau)\right)^{+} 1_{\left\{\max _{0 \leq u \leq \tau}\left(e^{-\Lambda_{n}(u)} S(u)\right)<h\right\}}\right] \\
& \geq \mathbf{E}_{0}\left[\liminf _{n}\left(e^{-r \tau-\alpha \Lambda_{n}(\tau)}\left(q-e^{-\Lambda_{n}(\tau)} S(\tau)\right)^{+} 1_{\left\{\max _{0 \leq u \leq \tau}\left(e^{-\Lambda_{n}(u)} S(u)\right)<h\right\}}\right)\right] \\
& \geq \mathbf{E}_{0}\left[e^{-r \tau-\alpha \Lambda(\tau)}\left(q-e^{-\Lambda(\tau)} S(\tau)\right)^{+} 1_{\left\{\max _{0 \leq u \leq \tau}\left(e^{-\Lambda(u)} S(u)\right)<h\right\}}\right]=R,
\end{aligned}
$$

by Fatou's Lemma, the Bounded Convergence Theorem, and the fact that we have

$$
\liminf _{n} 1_{\left\{\max _{0 \leq u \leq \tau}\left(e^{-\Lambda_{n}(u)} S(u)\right)<h\right\}} \geq 1_{\left\{\max _{0 \leq u \leq \tau}\left(e^{-\Lambda(u)} S(u)\right)<h\right\}} \cdot
$$

This is certainly true on $\left\{\max _{0 \leq u \leq \tau}\left(e^{-\Lambda(u)} S(u)\right) \geq h\right\}$; on the other hand, on the event $\left\{\max _{0 \leq u \leq \tau}\right.$ $\left.\left(e^{-\Lambda(u)} S(u)\right)<h\right\}$ we have $\max _{0 \leq u \leq \tau}\left(e^{-\Lambda_{n}(u)} S(u)\right)<h$ for all $n$ large enough, and (7.4) follows. 
Acknowledgement: We are indebted to Professor Steven Shreve for a talk at Columbia University on Uwe Wystup's dissertation, which was the inspiration for this work. We also want to thank Drs. Jakša Cvitanić, Mark Davis, Paavo Salminen and Qiang Zhang, for helpful discussions.

\section{References}

[1] Black, F. \& Scholes, M. (1973) The pricing of options and corporate liabilities. J. Political Economy 81, 637-659.

[2] Broadie, M., Cvitanić, J. \& Soner, M. (1998) Optimal replication of contingent claims under portfolio constraints. Review of Financial Studies, Vol. 11, No. 1, 59-79.

[3] Karatzas, I. (1996) Lectures on the Mathematics of Finance. CRM Monograph Series, Vol. 8, American Mathematical Society.

[4] Karatzas, I. \& Kou, S.G. (1998) Hedging American contingent claims with constrained portfolios. Finance and Stochastics 3, 215-258.

[5] Karatzas, I. \& Shreve, S. (1984) Connections between optimal stopping and singular stochastic control I: Monotone follower problems. SIAM J. Control \& Optimization 22, 856-877.

[6] Karatzas, I. \& Shreve, S. (1991) Brownian Motion and Stochastic Calculus. Second Edition, Springer-Verlag, New York.

[7] Karatzas, I. \& Shreve, S. (1998) Methods of Mathematical Finance. Springer-Verlag, New York.

[8] Merton, R.C. (1973) Theory of rational option pricing. Bell J. of Econom. \& Management Sci. 4, 141-183.

[9] Salminen, P. (1985) Optimal stopping of one-dimensional diffusions. Mathematische Nachrichten 124, 85-101.

[10] Wystup, U. (1997) Valuation of exotic options under short-selling constraints as a singular stochastic control problem. Doctoral Dissertation, Carnegie Mellon University. 